Supporting Information

\title{
Study on the synthesis of hydroxyapatite under highly alkaline conditions
}

\author{
Mauricio Córdova-Udaeta†, Yonggu Kim ${ }^{\dagger}$, Kazutaka Yasukawal,+, , Yasuhiro Katol,+, ,, , Toyohisa Fujita ${ }^{\ddagger}$, \\ Gjergj Dodbiba ${ }^{\dagger}$
}

\begin{abstract}
tDepartment of Systems Innovation, School Of Engineering, The University of Tokyo, 7-3-1 Hongo, Bunkyo-ku, 113-8656 Tokyo, Japan

‡School of Resources, Environment and Materials, Guangxi University, 100 Daxue Road, Nanning, Guangxi, 530004, P.R. China

IFrontier Research Center for Energy and Resources, Graduate School of Engineering, The University of Tokyo, 7-3-1 Hongo, Bunkyo-ku, 113-8656 Tokyo, Japan

TOcean Resources Research Center for Net Generation, Chiba Institute of Technology, 2-17-1 Tsudanuma, Narashino, Chiba, 275-0016, Japan

'Submarine Resources Research Center, Research Institute for Marine Resources Utilization, Japan Agency for Marine-earth Science and Technology, 2-15 Natsushima-cho, Yokosuka, Kanagawa, 237-0061, Japan
\end{abstract}



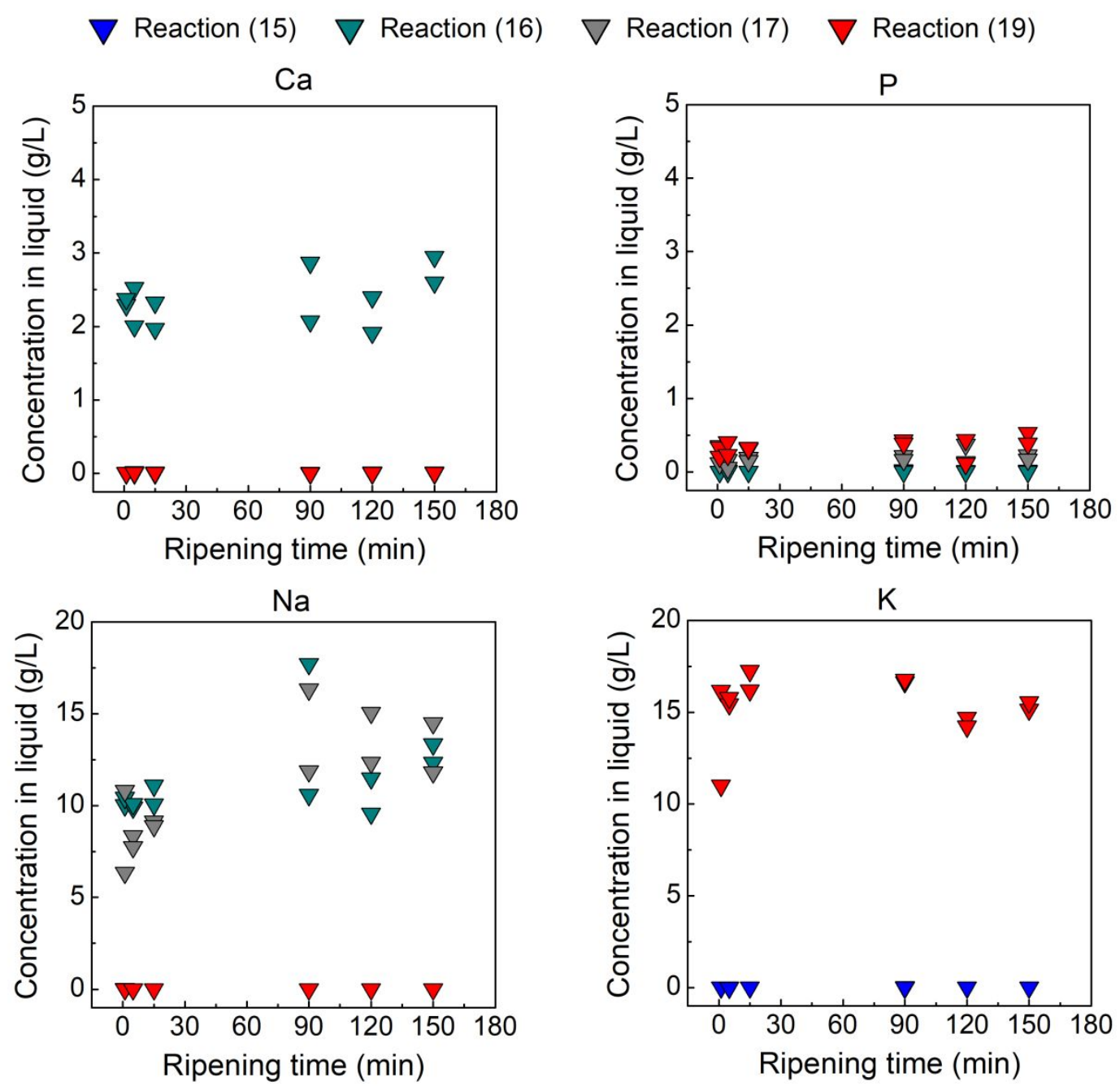

Supplementary Figure 1. Concentration of representative elements found in the liquid obtained after performing filtration on the HAP samples after precipitation. The concentration of the alkaline metal ions in the liquid is several times larger than $\mathrm{Ca}$ or $\mathrm{P}$. 
a)

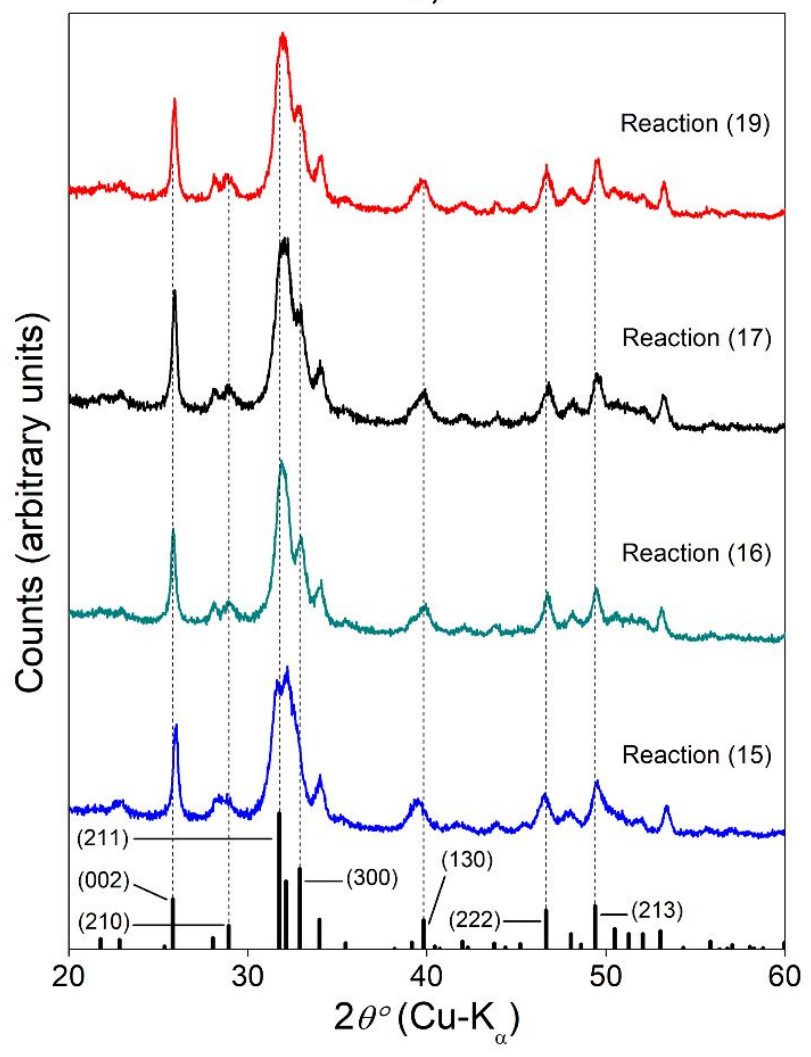

b)

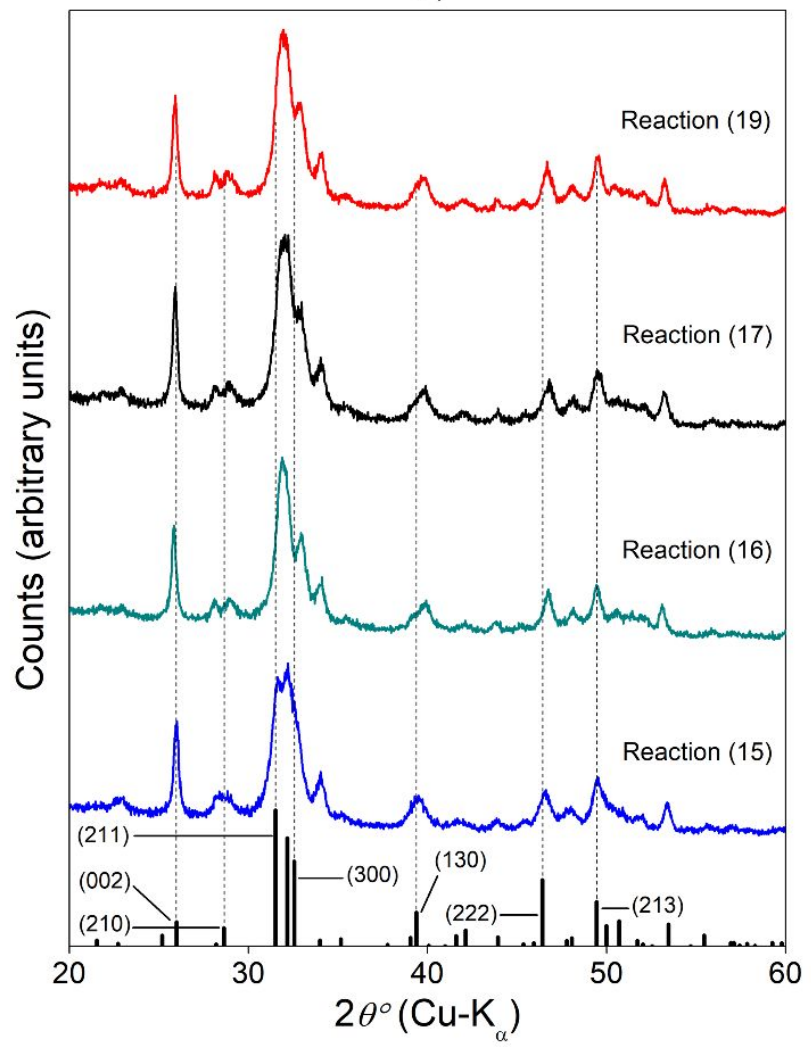

Supplementary Figure 2. Close-up of XRD results obtained for each reaction after a ripening time of $120 \mathrm{~min}$. The lines at the bottom correspond to the most relevant peaks of selected reference materials. a) Hydroxyapatite $\left(\mathrm{Ca}_{5}\left(\mathrm{PO}_{4}\right)_{3} \mathrm{OH}\right)$, ref. card: 01-082-2956, ICDD-PDF2 Database, 2019 ; b) Chlorapatite $\left(\mathrm{Ca}_{5}\left(\mathrm{PO}_{4}\right)_{3} \mathrm{Cl}\right)$, ref. card: 